ScIDice

\section{Posterior Subtenon Injection of 'Triamcinolone (PSTT) for Macular Edema in Various Retinal Conditions}

Research Article

Spoorti Mundinamani ${ }^{1}$, Pavana Acharya ${ }^{2 *}$, Kavitha Chikkanayakanahalli Venugopal ${ }^{3}$, Shruthi $\mathrm{K}^{4}$, Parvathy PS $^{5}$

${ }^{1}$ Post Graduate Student, Department of Ophthalmology, Hassan Institute of Medical Sciences, Hassan, Karnataka, India.

${ }^{2}$ Assistant Professor, Department of Ophthalmology, Hassan Institute of Medical Sciences, Hassan, Karnataka, India.

${ }^{3}$ Professor, Department of Ophthalmology, Hassan Institute of Medical Sciences, Hassan, Karnataka, India.

${ }^{4}$ Post Graduate Student, Department of Ophthalmology, Hassan Institute of Medical Sciences, Hassan, Karnataka, India.

${ }^{5}$ Department of Ophthalmology, Hassan Institute of Medical Sciences, Hassan, Karnataka, India.

\title{
Abstract
}

Importance: Cost effective way to resolve macular edema in few of the retinal conditions.

Background: To evaluate the effect on vision with Posterior Subtenon Triamcinolone acetonide injection for macular edema in various retinal conditions.

Design: Prospective type of interventional study.

Participants: 60 eyes of 54 patients with macular edema secondary to various retinal conditions. Of 60 eyes,12 BRVO, 23 CSME, 10 CRVO, 3 vitritis, 12 neuroretinitis.

Methods: Pre and post injection (of Posterior subtenon Triamcinolone), BCVA and IOP were evaluated at 1st week, 1 and 3 months.

Results: The mean age- 57.87 yrs, $40 \%$ were male and $60 \%$ were females. Pre injection Visual Acuity of $<6 / 60$ in $24,6 / 60-$ $6 / 24$ in 21 and $6 / 18-6 / 6$ in 15 patients. At least 2 line improvement in BCVA was noted in 28 eyes $(46 \%)$ at the end of study in which 11(39\%) BRVO, 3(10\%) CRVO, 8(33\%)CSME, 4(14\%) neuroretinitis and 2(7\%)vitritis. 3 patients developed IOP of $>21 \mathrm{mmHg}$ controlled medically. No other complications were observed.

Conclusions: Posterior Subtenon Triamcinolone acetonide is a cost effective modality of treatment for macular edema associated with retinal conditions with minimal complications.

Keywords: Posterior Subtenon Triamcinolone Acetonide; Macular Edema; Vein Occlusions; Diabetic Macular Edema: Vitritis; Neuroretintis.

\section{Introduction}

Macular edema occurs secondary to various disorders like retinal vein occlusions, diabetes, post cataract surgery, uveitis and neuroretinitis which lead to gross diminution of vision. Various treatment modalities have been tried to address the decreased vision due to macular edema. The results have varying success rates.

About $5-15 \%$ of eyes with BRVO develop macular edema over 1 year and the majority of patients with CRVO have signs of macular edema at presentation [1]. About $7 \%$ of people with diabetes may have DME [2]. Pseudophakic cystoid macular edema has been reported to be between $1 \%$ and $30 \%$ [3]. In post fever neuroretinitis, immunological reactions cause an increased vascular permeability of the retinal vasculature leading to retinal edema [4].

Resolution of macular edema can be long delayed. Cases that resolve spontaneously over a long interval will have neuroretinal or pigment epithelial scarring and atrophy. Hence, treatment of macular edema is essential to improve the vision [1].

Various treatment modalities for macular edema are focal/grid laser photocoagulation, Anti VEGF therapy (Pegaptinib sodium, Ranibizumab, Bevacizumab) and corticosteroids (either as intravitreal injection, intravitreal implants or subtenon injection).

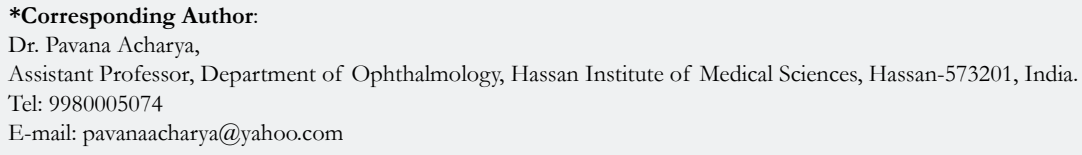

Citation: Spoorti Mundinamani, Pavana Acharya, Kavitha Chikkanayakanahalli Venugopal, Shruthi K, Parvathy PS. Posterior Subtenon Injection of Triamcinolone (PSTT) for Macular Edema in Various Retinal Conditions. Int J Ophthalmol Eye Res. 2020;8(3):429-433. doi: http://dx.doi.org/10.19070/2332-290X-2000087

Copyright: Pavana Acharya ${ }^{\circ}$ 2020. This is an open-access article distributed under the terms of the Creative Commons Attribution License, which permits unrestricted use, distribution and reproduction in any medium, provided the original author and source are credited. 
Triamcinolone acetonide is a glucocorticoid which is used as periocular injection in various ocular conditions like resistant anterior uveitis, intermediate uveitis, posterior uveitis, age related macular degeneration, macular edema due to vein occlusions (CRVO, BRVO), macular edema secondary to diabetes, post cataract surgery $[5,6]$. Triamcinolone-acetonide in addition to its anti-inflammatory effects, causes down-regulation of vascular endothelial growth factor (VEGF) [7]. In retinal disorders, it is used as a depot injection either through subtenon or intravitreally. Subtenon injections are less invasive and has less complications when compared to intravitreal injections [8]. The various complications of intravitreal injection which can be bypassed by using subtenon injections are vitreous haemorrhage, retinal detachment, vitreous detachment, endophthalmitis, high intraocular pressure. Subtenon corticosteroid is also cost effective compared to other modalities. In this study, we evaluate the efficacy of posterior subtenon injection of Triamcinolone acetonide injection in resolving macular edema in retinal vein occlusions, diabetic maculopathy, post uveitis and neuroretinitis.

\section{Materials and Methods}

A prospective interventional study was conducted in 60 eyes of 54 patients attending outpatient Department of Ophthalmology at HIMS teaching Hospital ,Hassan, Karnataka in the study period of December 2017 to September 2018.

Informed consent was taken from all patients included in the study.

The patients are included in the study by applying the following Inclusion and Exclusion criteria.

\section{Inclusion criteria}

Patient with macular edema secondary to vein occlusions, Diabetic maculopathy, uveitis, neuroretinitis.

\section{Exclusion criteria}

Following patients will be excluded from the study-

- Traumatic macular edema.

- Patients with decreased vision due to cataract, corneal pathology, other retinal disorders.
- All patients with intraocular pressure more than $21 \mathrm{mmHg}$.

- Patients with prior laser,intra vitreal or subtenon injections

\section{Methodology}

Patients fulfilling the inclusion criteria were recruited into the study. Patients underwent BCVA, anterior segment evaluation, dilatation and fundoscopy was done with indirect ophthalmoscopy with 20D lens. IOP measurement was done with Perkin's tonometer. Posterior Subtenon Triamcinolone Acetonide injection is given under local anaesthesia in operation theatre.

Under topical anaesthesia, injection of $0.5 \mathrm{ml}$ of $20 \mathrm{mg}$ of the drug using a sharp tipped 25-gauge needle that was inserted in the supratemporal quadrant of the eye, bevel of the needle facing towards the globe, and needle advanced to the hub to obtain adequate placement of the drug into the posterior sub tenon space. Following injection, topical antibiotic was instilled and eye was patched for 2 hours. Post procedure, patient was advised topical antibiotic (Moxifloxacin) and topical NSAIDS for two weeks and one month respectively.

\section{Follow up}

Patients were followed up at 1 week, 1 month, 3 months. During follow up BCVA,IOP and dilated fundus examination was done.

\section{Results}

The study was conducted on 60 eyes of 54 patients with macular edema secondary to various retinal conditions. Of 60 eyes,12 BRVO, 23 CSME, 10 CRVO, 3 vitritis, 12 neuroretinitis. The mean age in BRVO group was 52 yrs, CRVO group was 63 years, DME group was 55 years, Uveitis group was 39 years and Neuroretinitis group was 35 years. All the groups showed female dominance.

Two line improvement in the Snellan's visual acuity at the end of three months of follow up was considered as significant improvement.

The vision at pre- injection and post injection:- Pre- injection vision in BRVO group - 2 had $6 / 60,7$ had in the range of $6 / 60$ $-6 / 24,3 \mathrm{had}$ in the range of $6 / 18-6 / 6$. Post injection, $1 \mathrm{had}$ $<6 / 60,2 \mathrm{had}$ in the range of $6 / 60-6 / 24,9 \mathrm{had}$ in the range of $6 / 18-6 / 6$.

Figure 1. Mean Age in years in BRVO, CRVO, DME, Uveitis and Neuroretinitis groups.

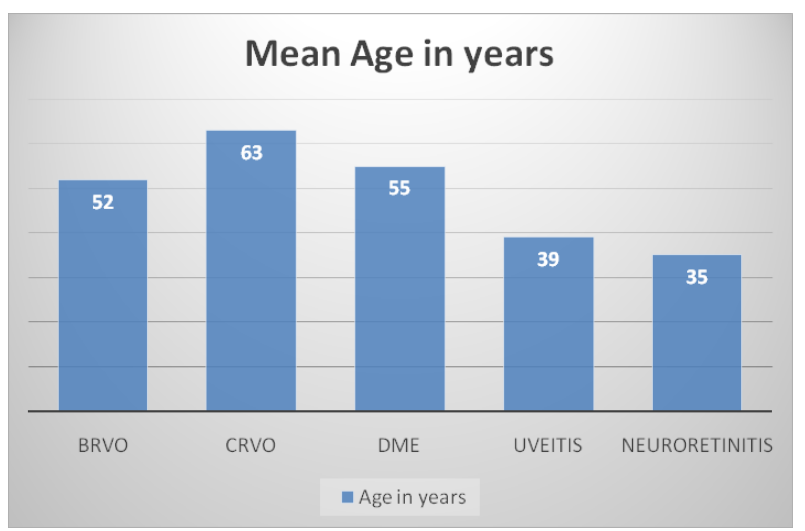


Figure 2. Gender distribution in BRVO, CRVO, DME, Uveitis and Neuroretinitis groups.

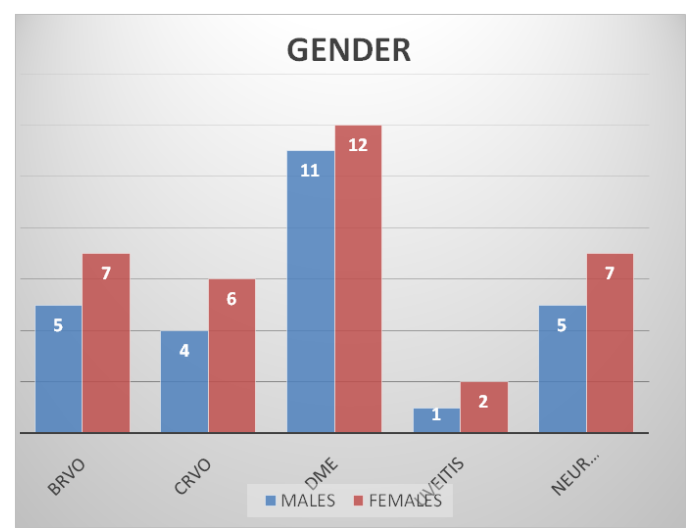

Table 1. Reference numbers with visual acuity in Snellan's visual acuity.

\begin{tabular}{|c|}
\hline $8-6 / 6-6 / 12$ \\
\hline $7-6 / 18$ \\
\hline $6-6 / 24$ \\
\hline $5-6 / 36$ \\
\hline $4-6 / 60$ \\
\hline $3-3 / 60$ \\
\hline $2-2 / 60$ \\
\hline $1-<1 / 60$ \\
\hline
\end{tabular}

Figure 3. No. of patients pre injection and post injection in BRVO group with their visual acuity represented in numbers as in table 1,11 out of 12 eyes show two line improvement.

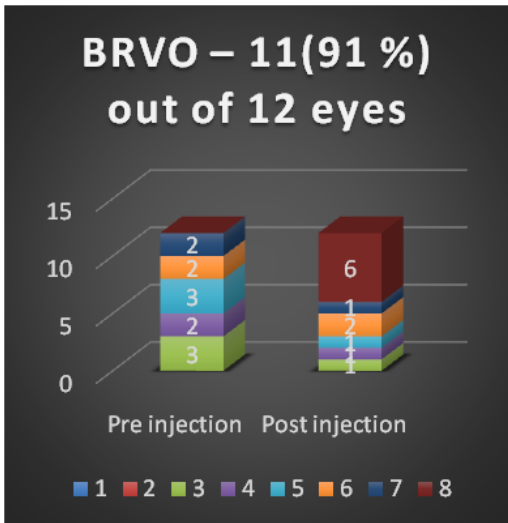

Pre- injection vision in CRVO group -5 had $6 / 60,3$ had in the range of $6 / 60-6 / 24,1$ had in the range of $6 / 18-6 / 6$. Post injection, $4 \mathrm{had}<6 / 60,3 \mathrm{had}$ in the range of $6 / 60-6 / 24,2$ had in the range of $6 / 18-6 / 6$.

Figure 4. No. of patients pre injection and post injection in CRVO group with their visual acuity represented in numbers as in table 1,3 out of 10 eyes show two line improvement.

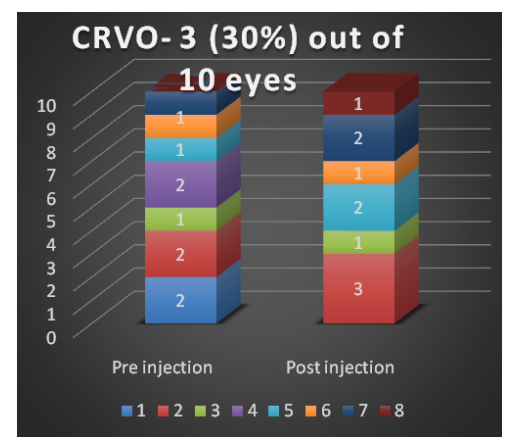

Pre- injection vision in DME group $-8 \mathrm{had}<6 / 60,13 \mathrm{had}$ in the range of $6 / 60-6 / 24,3$ had in the range of $6 / 18-6 / 6$. Post injection, $4 \mathrm{had}<6 / 60,14 \mathrm{had}$ in the range of $6 / 60-6 / 24,5$ had in the range of $6 / 18-6 / 6$ 
Figure 5. No. of patients pre injection and post injection in DME group with their visual acuity represented in numbers as in table 1,13 out of 23 eyes show two line improvement.

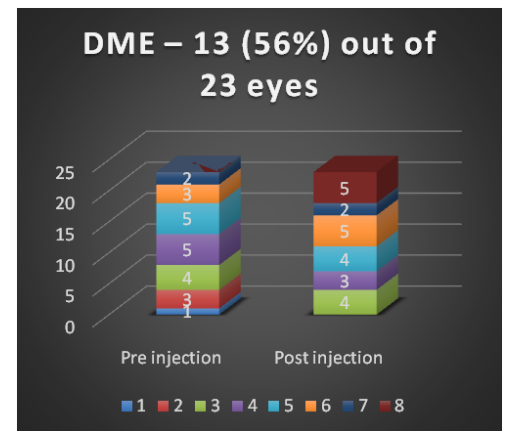

Pre- injection vision in neuroretinitis group $-2 \mathrm{had}<6 / 60,10 \mathrm{had}$ in the range of $6 / 60-6 / 24$. Post injection, 9 had in the range of $6 / 60-6 / 24,3$ had in the range of $6 / 18-6 / 6$

Figure 6. No. of patients pre injection and post injection in neuroretinitis group with their visual acuity represented in numbers as in table 1,4 out of 12 eyes show two line improvement.

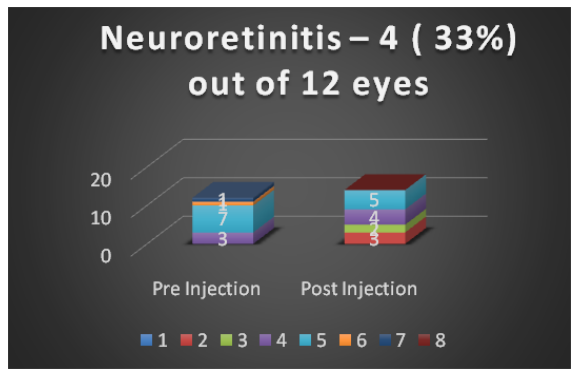

Pre - injection vision in vitritis group - 2 had 6/60,1 had 3/60. Post injection 2 had 6/24, 1 had 6/18

Figure 7. No. of patients pre injection and post injection in vitritis group with their visual acuity represented in numbers as in table 1,2 out of 3 eyes show two line improvement.

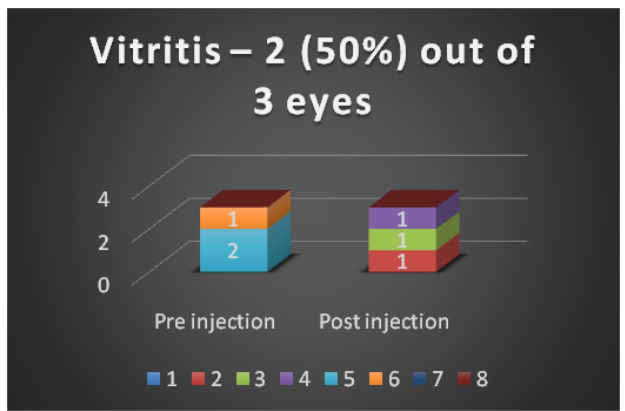

Thus, two line improvement in Snellan's visual acuity was seen in total of 28 eyes ( $46 \%$ ) of 60 eyes. Among them, 11 (39\%) were BRVO , 3 ( 10\%) were CRVO, 8 (33\%) were DME , 2 ( $7 \%$ ) were vitritis and $4(14 \%)$ were neuroretinitis.

Raised IOP ( $>21 \mathrm{mmHg}$ ) was noted in 4 eyes, one week after the injection which was controlled by the addition Timolol eye drops.

\section{Discussion}

Macular edema results from an increased vascular permeability mediated at least in part by upregulation of VEGF. Triamcinolone acetonide along with its anti inflammatory effect has been shown to inhibit the expression of VEGF and thus reduce macular edema in retinal vascular disease [8]. Early treatment of macular edema may be better for visual improvement before longstanding macular edema results in irreversible photoreceptor damage [9].

In our study, 3 out of 10 patients in CRVO group showed two line improvement. Lin et al., in a prospective, non-comparative study treated 18 eyes of 18 patients of severe CME with recent onset CRVO, with PSTT. They concluded that PSTT is effective in reversing CME and improving VA in recent-onset CRVO. They also recommended early treatment before long-standing macular edema results in irreversible photoreceptor damage [9].

In our study, $11(90 \%)$ out of 12 BRVO patients had a two line improvement post PSTT, similar results were seen in a study by Nil et al. who evaluated 24 eyes with macular edema secondary to RVO. All the eyes were treated with PSTT. 58.3\% showed increased BCVA with no major complications. They concluded that PSTT $T$ was an effective therapeutic method to treat macular edema associated with RVO [10].

In our study, two out of three patients with vitritis showed two line improvement.In a study by Lanco Daflon et al ,nearly $80 \%$ of the treated eyes with vitritis showed an improvement of at least 2 Snellen lines, and $20 \%$ of all eyes had an improvement of more than 5 Snellen lines at long-term follow-up [11]. The same was also studied by Helm et al., [12] 
In our study, 8 (34\%) out of 23 eyes with DME showed two line improvement. PSTT in DME eyes was studied by Ozdek et al to evaluate retro prospectively the clinical consequences of posterior subtenon (PSTT) and intravitreal (IVT) triamcinolone acetonide injections in diabetic macular edema (DME). They concluded that both PSTT and IVT injections caused a significant increase in visual acuity [13].

In our study, $4(14 \%)$ out of 12 eyes with neuroretinitis showed two line improvement.The immunological nature of post fever neuroretinitis and its favourable response to systemic steroids is reported by sreelatha viswanath et al., [14].

Rise in intraocular pressure following posterior subtenon injection and intra vitreal injection of triamcinolone acetonide is a complication reported in various studies. However the incidence in IOP rise is less in PST than in intra vitreal injection [15]. We found no significant elevation in intraocular tension following the procedure similar to the results of Bui Quocket et al., [16]

\section{Conclusion}

Posterior subtenon injection with triamcinolone acetonide can be considered as a cost effective and safe alternate choice for treating macular edema secondary to vein occlusions, diabetic macular edema, parsplanitis, neuroretinitis without any risk of complications.

\section{Ethical Considerations}

Ethical clearance was obtained prior to the study from Institutional Ethics Committee, HIMS, Hassan with reference no. IEC/ HIMS/014/30-05-2018. The study has been performed in accordance with the ethical standards laid down in an appropriate version of the Declaration of Helsinki.

\section{References}

[1]. McIntosh RL, Rogers SL, Lim L, Cheung N, Wang JJ, Mitchell P, et al. Natural history of central retinal vein occlusion: an evidence-based systematic review. Ophthalmology. 2010 Jun 1; 117(6): 1113-23.
[2]. Lee R, Wong TY, Sabanayagam C. Epidemiology of diabetic retinopathy, diabetic macular edema and related vision loss. Eye and vision. 2015 Sep 30; 2(1): 17. PMID: 26605370.

[3]. Grzybowski A, Sikorski BL, Ascaso FJ, Huerva V. Pseudophakic cystoid macular edema: update 2016. Clinical interventions in aging. 2016; 11: 1221-1229. PMID: 27672316.

[4]. Purvin, Valerie MD, Sundaram, Seema MD, Kawasaki, Aki MD. Neuroretinitis: Review of the Literature and New Observations. J Neuroophthalmol 2011; 31(1): 58-68. PMID: 21317731.

[5]. Greenberg PB, Martidis A, Rogers AH, Duker JS, Reichel E. Intravitreal triamcinolone acetonide for macular oedema due to central retinal vein occlusion. British Journal of Ophthalmology. 2002 Feb 1; 86(2): 247-8.

[6]. Ip MS, Gottlieb JL, Kahana A, Ingrid U Scott, Michael M Altaweel, Barbara A Blodi, et al. Intravitreal triamcinolone for the treatment of macular edema associated with central retinal vein occlusion. Arch Ophthalmol. 2004; 122: 1131-1136. PMID: 15302652.

[7]. Nauck M, Roth M, Tamm M, O Eickelberg, H Wieland, P Stulz, et al. Induction of vascular endothelial growth factor by platelet-activating factor and platelet-derived growth factor is down regulated by corticosteroids. Am J Resp Cell Mol Biol. 1997; 16: 398-406. PMID: 9115750.

[8]. Wakabayashi T, Okada AA, Morimura Y, Kojima E, Asano Y, Hirakata A, et al. Trans-tenon retrobulbar triamcinolone infusion for chronic macular edema in central and branch retinal vein occlusion. Retina. 2004 Dec 1; 24(6): 964-7.

[9]. Lin JM, Chiu YT, HUNG T, Tsai YY. Early treatment of severe cystoid macular edema in central retinal vein occlusion with posterior sub-tenon triamcinolone acetonide. Retina. 2007; 27(2): 180-9. PMID: 17290200.

[10]. Nil Rem, Mehmet, Cenk, Zeliha, Gursel Emin. Posterior Subtenon Triamcinolone Acetonide in the Treatment of Macular Edema Secondary to the Retinal Vein. Occlusion Journal of Retina-Vitreous. 2004; 12(3): 180-83.

[11]. Marina Lafranco Dafflon, Van Tao Tran, Y Guex-Crosier, Carl P Herbort. Posterior sub-Tenon's steroid injections for the treatment of posterior ocular inflammation: indications, efficacy and side effects. Graefe's Archive for Clinical and Experimental Ophthalmology. 1999; 237(4): 289-295. PMID: 10208261.

[12]. Helm JH, Holland GN. The effects of posterior sub tenon injection of triamcinolone acetonide in patients with intermediate uveitis. Am J Ophthalmol. 1995; 120: 55-64. PMID: 7611330.

[13]. Özdek Ş, Bahçeci UA, Gürelik G, Hasanreisoğlu B. Posterior subtenon and intravitreal triamcinolone acetonide for diabetic macular edema. Journal of diabetes and its complications. 2006 Jul 1; 20(4): 246-51. PMID: 16798476.

[14]. Srilatha Vishwanath, Kalpana Badami, KS Sriprakash, BL Sujatha, SD Shashidhar, YD Shilpa. Post-fever retinitis: a single center experience from south India. Int Ophthalmol. 2014; 34: 851-857. PMID: 24362635.

[15]. Iwao K, Inatani M, Kawaji T, Koga T, Mawatari Y, Tanihara H. Frequency and risk factors for intraocular pressure elevation after posterior sub-Tenon capsule triamcinolone acetonide injection. Journal of Glaucoma. 2007; 16(2): 251-6. PMID: 17473740.

[16]. Bui Quoc E, Bodaghi B, Adam R, Burtin T, Cassoux N, Dreifuss S, et al. Intraocular pressure elevation after subtenon injection of triamcinolone acetonide during uveitis. J Fr Ophtalmol 2002; 25(10): 1048-56. PMID: 12527830 . 